\title{
Reversible Capacitance Change of Nematic Liquid Crystal Cell Doped with Semiconductor CdSe/ZnS Quantum Dots
}

\author{
E.A. Konshina, I.F. Galin ", E.O. Gavrish \\ National Research University of Information Technologies, Mechanics and Optics \\ *Corresponding Author: ildar.f.galin@gmail.com
}

Copyright (C) 2014 Horizon Research Publishing All rights reserved.

\begin{abstract}
Electrical characteristics of the nematic LC cells with $3.5 \mathrm{~nm}$ semiconductor $\mathrm{CdSe} / \mathrm{ZnS}$ quantum dots (QDs) were investigated. We observed variation of the LC cell capacitance with QDs concentration about 0.18 wt. \%. The capacitance grown to three orders of magnitude at primary measuring under voltages below the Fredericks threshold and then it dropped. Repeated tests shown that the capacitance maximum of dependence decreased and disappeared after repeated measurements of the LC cell. The maximum arose again after applying to the LC cell of the alternating-current electric field with voltage of $30 \mathrm{~V}$ at a frequency of $1 \mathrm{kHz}$ for 10 minutes. Changing of the capacitance was observed in the result of mobile ions interactions of liquid crystal with QDs in the electric field. That contributed to an aggregation and moving charged nanoparticles in the direction of substrates of LC cell as well as their reverse motion to a centre under action of applied voltage onto the LC cell.
\end{abstract}

Keywords Nematic Liquid Crystal; CdSe/ZnS Quantum Dots; Capacitance

\section{Introduction}

Semiconductor quantum dots (QDs) with the unique electronic properties are one of the well-studied classes of nanoparticles. QDs are a new promising platform for variation properties of optical, electro-optical properties and orientation of liquid crystalline materials [1]. Doping liquid crystals (LCs) by semiconductor QDs opens up great possibilities for parameter variation of LC photonic devices. For example, photorefractive responses may be enhanced if CdSe QDs add to nematic liquid crystal (NLC) cells, thus enabling real time image processing application unlike other doped systems. The switching voltage required for electro-optical modulation of NLCs with these CdSe QDs is considerably lower than undoped NLC. Such low-switching voltage and photorefractive NLC's are promising candidates for electro and nonlinear-optical image processing and switching application [2]. Variation of band gap energy semiconductor QDs influences on the optical properties of liquid crystal structures [3]. Remarkable enhancement in the photoluminescence intensity and a significant shift of the emission band of ferroelectric liquid crystals (FLC) materials are observed by doping with a suitable amount of QDs [4].

The implementation of any electro-optic effect in liquid crystals is not possible without the application of an electric field. The motion of charged nanoparticles under $\mathrm{dc}$ electrical field action was observed in a hybrid LC cell doped by nanoparticles. An orientation of nematic liquid crystal with solid nanoparticles was controlled by the optically "hidden" electrophoretic effect [5]. The studies of electric-field-induced dynamics of colloids in a nematic cell were shown that, solid particles in the nematic bulk create director distortions of dipolar type. Elastic repulsion from the walls keeps the particles in the middle of cell. The alternating-current (AC) electric field reorients the dipoles and lifts them to top or bottom, depending on dipole orientation. Near the walls of cell, the colloids are carried along two antiparallel horizontal directions by nematic backflow [6]. Mechanisms of interaction of nanoparticles with LCs molecule are not quite clear today, but a capture of mobile ions into LCs exercise a significant influence on it [7-9]. When a voltage is applied to LC cells, ions begin to move in the direction to electrodes in line with charge permutation. Dielectric nanoparticles polarized under an external electric field have the ability to decrease the ions number in the LC cell due to capturing and holding ions of opposite sign, and thus suppresses the undesired screening effect and contributes to a reduction of the threshold voltage $[10,11]$. The effective method of reducing mobile ions in LC cell with a silica nanoparticle ( $\mathrm{SN}$ ) was demonstrated recently using AC high-voltage pulse treatment. In this method, the director distortion in LCs near the substrate creates a lifting force that moves the SNs toward the substrate. The SNs and mobile ions are adsorbed onto the substrates, thereby significantly decreasing cell ion density [12]. Decrease of the Fredericks threshold is the result of lowering the elastic coefficient $K_{11}$ for the splay deformation nematic LCs with QDs and the effective dielectric constant owing to changing of an order parameter $[10,11,13]$. Doping QDs into FLC caused the sign reversal of dielectric 
anisotropy and the reverse switching of FLC [14]. Studies of the properties of liquid crystals with QDs for understanding the interaction between the host LC molecules and nanoparticles are topical issue.

We observed decline of the phase delay, the effective dielectric constant and the threshold voltage with increasing concentration from $\sim 0.1$ to $\sim 0.2$ wt. \% CdSe/ZnS QDs in the nematic LC [13]. Comparison of the electrical characteristics of the LC cells showed a variation of capacitance and resistance with increasing of $\mathrm{CdSe} / \mathrm{ZnS}$ concentration comparing to the LC cells without QDs. Note that characteristics of the nematic LC cells with concentrations QDs about 0.2 wt. \% were unstable and the effective dielectric constant reduced. A probable cause of the LC cells parameter instability is allocations change of nanoparticles in the LC matrix in the result of their drift. In this work we compared experimental dependence of capacitance and resistance on voltage of two LC cells with CdSe/ZnS QDs with concentration about 0.1 and $0.18 \mathrm{wt}$. \% to look variation of electrical properties in time and in the result of AC voltage action.

\section{Materials and Methods}

The experiments were performed on electrically controlled LC cells with a diameter of $35 \mathrm{~mm}$. The nematic LC of ZhK-1282 type (NIOPIK, Moscow) with positive dielectric anisotropy was used for cells fabrication. We used the colloidal CdSe/ZnS semiconductor QDs representing composite nanoparticles of the core/shell type. Each QD consists of CdSe core with a diameter of $\sim 3.5 \mathrm{~nm}$ (fundamental absorption band of CdSe is peaked at $\sim 570 \mathrm{~nm}$ ) and $\mathrm{ZnS}$ passivating shell covered by a layer of trioctylphosphine oxide. The QDs were synthesized at the Institute for Physicochemical Problems (Minsk) using a method described in [15]. Each empty LC cell was manufactured with two flat glass substrates coated with ITO electrodes and a-C:H alignment layers for planar orientation [16]. A cell gap about $17 \mu \mathrm{m}$ is fixed out by thin Teflon films as spacers. The LC mixtures with nanoparticles were prepared by adding QDs into LC in its nematic phase. Prior to filling the cells, the suspensions of QDs in LC were thoroughly treated in an ultrasonic bath for 1 hour, since otherwise nanoparticles poorly mixed with LC and tended to precipitate. The LC/QDs mixture in the nematic phase was introduced into empty cell by capillary action. All cells were characterized by homogeneous orientation of the LC director field, which was observed in a polarization microscope.

The LC cells capacitance and resistance were measured using a special computer-controlled electric circuit on a sinusoidal voltage with a frequency of $1 \mathrm{kHz}$ and amplitude within $0.3-10 \mathrm{~V}$. All electrical measurements were performed at room temperature.

\section{Results and Discussion}

Dependences of capacitance and resistance of the LC cell with the concentration about 0.1 wt. \% is shown in Figure 1. Dependences of capacitance and resistance of the LC cell with the concentration about wt. \% is shown in Figure 1. The dependence capacitance -voltage is in accordance with dependence for a LC cell without QDs. Characteristics of the LC cell were stable and we did not observe variation of them in time. The threshold of electro-optic effect for the LC cells with a-C:H alignment layer is about $2 \mathrm{~V}$ [16]. Ions gathered near the surface of the alignment layer create a space charge field. A number of mobile ions in LC bulk can reduce in the result of capture their by nanoparticles. Lowering of a space charge and screening of applied voltage lead to decrease of threshold voltage. The QDs doping reduced the threshold voltage up to $\sim 1 \mathrm{~V}$ as can see in Figure 1.

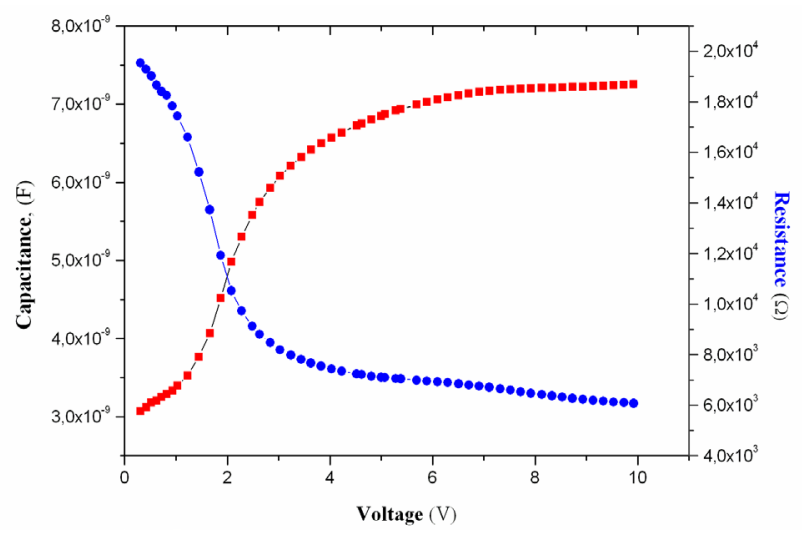

Figure 1. Dependences of capacitance and resistance of the LC cell doped with 0.1 wt. $\%$ QDs.

We observed an unusual change of the capacitance-voltage dependence of the LC cells with the QDs concentration about $~ 0.18$ wt. \% as are shown in Figure 2. This dependence sharply rises by three orders of magnitude in the range of voltages below the Fredericks threshold for the LC cell as compared to the dependence in Figure 1 and then drops. The curve of resistance-voltage in Figure 2 lays lower the curve in Figure 1. This implies lowering of LC cell resistance with increasing of semiconductor QDs concentration. Surface states of QDs are not rule out completely in spite of CdSe core of the QDs is surrounded by $\mathrm{ZnS}$ semiconductor material with a wider gap. These states may be traps for mobile ions. The QDs polarization occurs under voltages is below the threshold voltage of the LC cell. We suggest that an abnormal increase in LC cell capacitance under applying voltage (Figure 2) may be in the result of mobile ions capturing. That affects the dielectric constant of the suspension. The polarization of liquid crystal molecules and their reorienting occurs when the applied voltage more than the threshold voltage of the cell. Therefore the decline of the capacitance at curve in Figure 2 and then its subsequent rise are possible due to changes in the LC matrix structure associated with the reorientation of dipoles of the $\mathrm{LC}$ molecules in the electric field. 


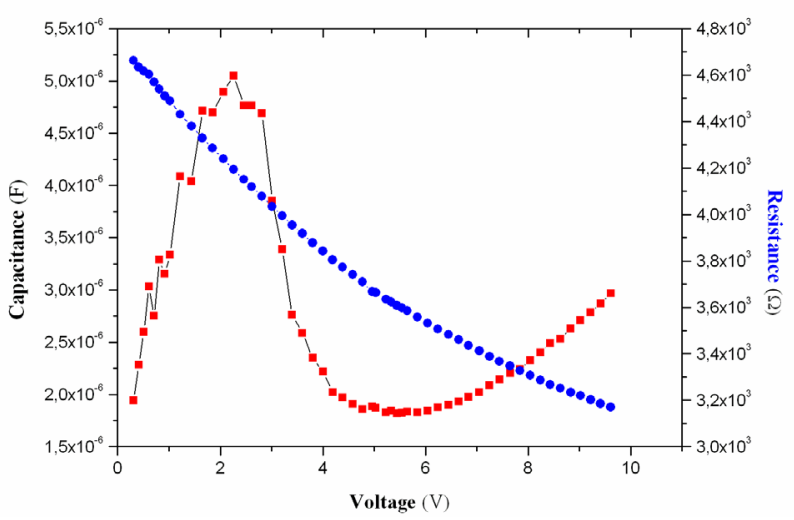

Figure 2. Dependences of capacitance and resistance of the LC cell doped with $0.18 \%$ QDs.

The capacitance of the LC cell with a concentration of about $0.18 \%$ QDs was tested few times during one year. Figure 3 illustrates changing the capacitance of the LC cell: a - initial, b - two month later, c - one year later. We can see that the capacitance of the LC cell decreased as compared to initial during two months (curve b, Figure 3). The capacitance dependence decreased by three orders of magnitude (curve c, Figure 3) the year later. The degradation of the LC cell capacitance might be explained by charging of nanoparticles distribution in the LC matrix. Formation of aggregates from charged solid nanoparticles and their movement towards the LC cell substrates under the influence of gravitational forces lead to compositional disorder. Concentration of QDs in the LC bulk became lower than near the cell substrates and dielectric constant of the suspension reduced.

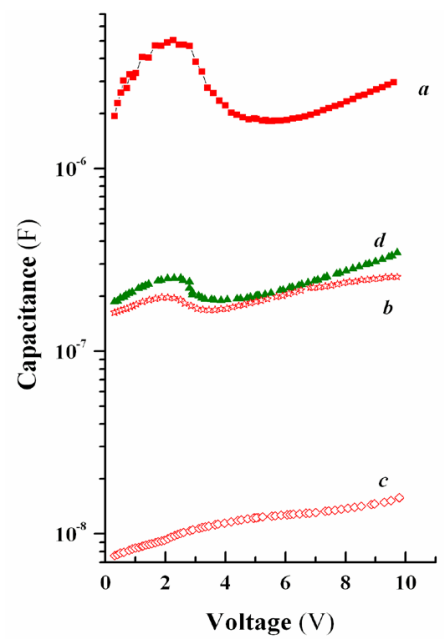

Figure 3. Variation capacitance of the LC cell with concentration about 0,18 wt.\% QDs: the initial measurement $(a)$, repeated measurements after two months $(b)$ and after one year $(c)$, after the application to the cell of an electric field with an amplitude of voltage $30 \mathrm{~V}$ and frequency of $1 \mathrm{kHz}$ for 10 minutes (d).

Then we measured the dependence of capacitance after applying the LC cell ac electric field with voltage of $30 \mathrm{~V}$ and a frequency of $1 \mathrm{kHz}$ for 10 minute. We observed the inverse process of capacitance increasing again. The curve $d$ obtained after applying an electric field to the LC cell coincides with the curve $\mathrm{b}$ as is evident in Figure 3. Molecules of the liquid crystal near the surface keep their orientation, due to the strong adhesion energy with the a-C:H orienting layer. Molecules in the LC bulk change their orientation on vertical, during exposure to the ac electric field. Sure as changing of the LC matrix structure contributes to moving charged nanoparticles in the direction from substrates to middle of LC cell.

The mechanism change dielectric properties of the suspension with concentration of 0.18 wt. \% QDs in electric field may be associated with few competitive processes such as the capture of mobile ions on surface states of QDs in the range voltages bellow Fredericks threshold, a formation of aggregates of the charged nanoparticles or clusters and their moving with rise of voltage. The diffusion of aggregates of QDs takes place in the lack of electric field under gravitational force.

The maximum on curve of capacitance in the range voltages bellow Fredericks threshold gradually disappear because of movement of the ODs clusters and mobile ions to interface of the LC with cell substrates. When ac electric field with high voltage is applied to the LC cell a destruction of the clusters on separate charged nanoparticles takes place and they can move to center of cell. The forma of capacitance curve in the result alters again in the range voltages bellow Fredericks threshold. The analysis of capacitance variation of the LC cell with the higher concentration of $\mathrm{CdSe} / \mathrm{ZnS}$ QDs shown possibility reversible change of capacitance with using high voltage

\section{Conclusion}

The electrical characteristics of the nematic LC cells containing $3.5 \mathrm{~nm}$ semiconductor $\mathrm{CdSe} / \mathrm{ZnS}$ QDs were studied. The dependences of capacitance for two LC cells with concentration about 0.1 and 0.18 wt. \% QDs were compared. The unusual variation of dependence of capacitance on voltage of the LC cell with a higher concentration of QDs was observed. The capacitance rise by three orders of magnitude originally at the voltages below the Fredericks threshold. The peak of capacitance on the curve decreased gradually and vanished completely. The reversible process of the capacitance variation of the LC cell was observed under applying ac electric field with voltage 30 $\mathrm{V}$ at a frequency of $1 \mathrm{kHz}$ for 10 minutes.

The change of capacitance is associated with alter of dielectric constant of the LC suspension with QDs. The significant increase in the cell capacity become at the initial time due to the polarization of the QDs and the capture of mobile ions on the surface states of the QDs. Formation of dipoles of the LC molecules begins at the voltage more $2 \mathrm{~V}$, they are oriented along the vector of the applied electric field and position of LC director becomes vertical. Some of the ions leave the QDs traps with rise of voltage and the capacitance change take place in a result of increasing 
component of the dielectric constant of the LC parallel of the director. The variation of capacitance LC cells with a higher concentration of the QDs is related to the interaction between the QDs and mobile ions with variation of voltage of electric field which effect on orientation of the liquid crystal molecules simultaneously. Lowering capacitance of the LC cell observed under subsequent testing, which was involved with a change of homogeneity of the QDs distribution in LC matrix in the result of QDs aggregates and movement to the interface. Vertical reorientation director in the ac electric field facilitates reciprocal movement of mobile ions as well as charged QDs in the LC cell. The unusual dependence of capacitance- voltage of the LC cell with a higher concentration QDs reset under action electric field. That result testifies about reversible change dielectric constant and capacitance of LC materials with semiconductor QDs.

The mechanism change dielectric properties of the suspension with concentration of $0.18 \mathrm{wt} \%$ QDs in electric field may be associated with few competitive processes such as the capture of mobile ions on surface states of QDs in the range voltages bellow Fredericks threshold, a formation of aggregates of the charged nanoparticles or clusters and their moving with rise of voltage. The diffusion of aggregates of QDs takes place in the lack of electric field under gravitational force. The results of this study should promote understanding influence semiconductor QDs doping on dielectric properties of liquid crystals and may be useful for the development of optoelectronic devices on base liquid crystals with semiconductor QDs the near future.

\section{Acknowledgements}

This work was supported by Saint-Petersburg National Research University of Information Technologies as perspective investigations.

\section{REFERENCES}

[1] Mirzaei J, M. Reznikov J and Hegmann T: Quantum dots as liquid crystal dopants. J. Mater. Chem. 2012, 22: 22350-22365.

[2] Khoo IC, Williams YZ, Lewis B and Mallouk T: Photorefractive CdSe and gold nanowire-doped liquid crystals and polymer-dispersed-liquid-crystal photonic crystals. Mol. Cryst. Liq. Cryst. 2006, 446: 233-244.

[3] Anczykowska A, Bartkiewicz S, Nyk M and Mysliwiec J: Study of semiconductor quantum dots influence on photorefractivity of liquid crystals. Appl. Phys. Lett. 2012,
101: 101107(1-4).

[4] Kumar A, Tripathi S, Deshmukh A D, Haranath D, Singh P and Biradar AM: Time evolution photoluminescence studies of quantum dot doped ferroelectric liquid crystals. J. Phys. D: Appl. Phys. 2013, 46: 195302 (1-7).

[5] Sikharulidze D: Nanoparticles: An approach to controlling an electro-optical behavior of nematic liquid crystals. Appl. Phys. Lett. 2005, 86: 033507(1-3).

[6] Pishnyak O, Tang S, Kelly J, Shiyanovskii S, and Lavrentovich O: Levitation, Lift, and Bidirectional Motion of Colloidal Particles in an Electrically Driven Nematic Liquid Crystal Phys. Rev. Lett. 2007, 99: 127802 (1-4).

[7] Ha Y-S, Kim H-J, Park H-G and Seo D-S: Enhancement of electro-optic properties in liquid crystal devices via titanium nanoparticle doping. Optics Express. 2012, 20: 6448-6455.

[8] Chen P-S, Huang C-C, Liu Y-W and Chao C-Y: Effect of insulating-nanoparticles addition on ion current and voltage-holding ratio in nematic liquid crystal cells. Appl. Phys. Lett. 2007, 90: 211111 (1-3).

[9] Chen W-T, Chen P-S and Chao C-Y: Effect of doped insulating nanoparticles on the electro-optical characteristics of nematic liquid crystals. Jpn. J. of Appl. Phys. 2009, 48: $015006(1-5)$.

[10] Zhang T, Zhong $\mathrm{Ch}, \mathrm{Xu}$ J: CdS nanoparticle-doped liquid crystal displays showing low threshold voltage. Jpn. J. of Appl. Phys. 2009, 48: 055002 (1-6).

[11] Kinkead B and Hegmann T: Effects of size, capping agent and concentration of $\mathrm{CdSe}$ and $\mathrm{CdTe}$ quantum dots doped into a nematic liquid crystal on the optical and electro-optic properties of the final colloidal liquid crystal mixture. $J$. Mater. Chem. 2010, 20:448-458.

[12] Liao S-W, Hsieh C-T, Kuo C-C and Huang C-Y: Voltage-assisted ion reduction in liquid crystal-silica nanoparticle dispersions. Appl. Phys. Lett. 2012, 101, 161906 (1-4).

[13] Konshina EA, Gavrish EO, Orlova AO and Artem'ev MV. Effect of dispersed CdSe/ZnS quantum dots on optical and electrical characteristics of nematic liquid crystal cells. Tech. Phys. Lett. 2011, 37: 1011-1014.

[14] Kumar A, Silotia P, and Biradar AM: Sign reversal of dielectric anisotropy of ferroelectric liquid crystals doped with cadmium telluride quantum dots. Appl. Phys. Lett. 2011, 99: 072902 (1-3).

[15] Dabbousi BO, Rodriguez-Viejo J, Mikulec FV, Heine JR, Mattoussi H, Ober R, Jensen KF and Bawend M G: (CdSe)ZnS Core-Shell Quantum Dots: Synthesis and Characterization of a Size Series of Highly Luminescent Nanocrystallites. J. Phys. Chem. B. 1977, 101: 9463-9475.

[16] Konshina EA and Gavrish EO: Screening Effect of a-C:H Alignment Layer in Nematic Liquid Crystal Cell. Tech. Phys. Lett. 2011, 37: 455-457. 